\title{
Structuring flexibility: the potential good, bad and ugly in standardisation of handovers
}

\section{E S Patterson}

Few strategies routinely used during shift change handovers in high-reliability organisations (HROs) are used in healthcare. ${ }^{1}$ The findings from the paper in this issue by Borowitz et $a l^{2}$ (see page 6) add to the growing empirical evidence that patient handovers in all healthcare settings are highly variable in content and process. Similar to other highly respected healthcare researchers, the authors recommend reducing this variability, in this case by standardising sign-out protocols. However, similar to other human factors researchers, I am concerned about the potential unintended consequences from this well-intentioned approach.

A natural experiment is already underway in the USA to standardise patient handovers. This experiment will likely be shaped by these repeating patterns from human factors research in complex sociotechnical settings:

- all decisions require making trade-offs on competing goals;

- imposing a simple standard on a complex process does not result in simplicity;

- local actors must tailor a distant supervisor's plan to the context;

- people adapt procedures over time in response to feedback;

- communication is not a (macrocognitive) function, but rather a means to achieve multiple functions in necessarily distributed work;

- high-performing teams communicate less and more proactively (more push than pull) than low performing teams.

In the context of handover standardisation for patient care, this natural experiment is predicted to have positive impacts as well as negative, unintended consequences-in other words, the good, the bad, and the ugly.

Correspondence to: E S Patterson, Getting at Patient Safety (GAPS) Center, Veterans Administration, Institute for Ergonomics, Ohio State University, Columbus, Ohio, USA; patterson.150@osu.edu

\section{THE GOOD}

Communication is required to safely transfer responsibility and authority for patient care during a handover. Standardisation reduces the cost of communication because:

- the "rules" for interaction do not need to be negotiated (including the function, process, content, timing, and who is directly or indirectly included in the conversation);

- no information on a topic (usually) implies that there is nothing worthy of mention on that topic;

- information can be conveyed more efficiently and with higher reliability.

Perhaps more importantly, the process of standardisation provides a window of opportunity to restructure how work is normatively conducted. Technically, a new anchor for normative behaviour is set using the "anchor and adjust" strategy. The "adjust" term emphasises the need for flexibility within the structure to tailor the plan to a specific context, support exception handling for non-routine cases, and enable adaptation to feedback about priorities when making tradeoffs.

As many have observed, healthcare is a rapidly moving target given anticipated shifts in demographics, costs, technology, international competition and the role of patients in managing their own care. Room for change to new anchors needs to be built into standards. Based on experiences in other domains, improvements in interdisciplinary coordination might be a key leverage point in radically improving healthcare delivery. Redesigning handover processes could be a first step in a wedge of activity to improve coordination. Several organisations are already experimenting with new coordination models, including enabling shared decision making with non-doctors, instituting collaborative cross-checking to increase system resilience, including across authority gradients, ${ }^{3}$ using information technology to make work more observable to team members ${ }^{4}$ and increasing access to specialised off-site expertise via telemedicine (eg, the electronic intensive care unit).

\section{THE BAD}

A primary focus for patient handover standardisation has been ordering the content of the verbal update. Unfortunately, with the possible exception of nuclear submarine handovers, no HRO has yet been found to use a structured verbal update during a handover transition. In addition, data elements are notably absent during handover updates. ${ }^{5}$ Most likely, this is explained by the use of "most important first" as an ordering heuristic for topics in the verbal update. In addition, space shuttle mission controllers are reportedly trained to "efficiently communicate a bottom line and details when asked". Under data overload conditions in which a massive amount of data is theoretically available but pragmatically impossible to review, the verbal update is used to help the incoming practitioner quickly get a "story" of the situation (the technical terms for the macrocognitive functions supported by a handover update are problem recognition, analysis, sensemaking, and planning), which is not easily conveyed in a structured (checklist) format. Finally, ordering by "most important first" reduces the likelihood that interruptions will occur prior to finishing the verbal update. If content ordering becomes the primary focus during standardisation efforts, it is possible that exchanging paperwork will begin to substitute for verbal updates since that will ensure that information is reliably ordered in the desired way and/or "covert" verbal updates will occur that highlight the most important, informative aspects in a checklist.

Another recommendation for patient handover has been the inclusion of readbacks. Although readbacks are used prevalently for specific purposes such as verifying the correct receipt of a planned altitude change, they have never been observed during HRO handovers. One explanation is that, even with readbacks, verbal communications are more prone to erroneous information exchanges than written communication. Likely to meet the same intent as readbacks, written logs or report sheets are recommended for handovers in process operations, and we observed them to be routinely used prior to and/or following handover updates in most HROs. In some settings, including space shuttle mission control, logs contained a short summary that was written 
explicitly to support the handover process. Since it is hard to know what the equivalent of a "log" is in most healthcare settings, the prediction is that new paperwork will be created that will capture information into a written format using a readback that will then be exchanged during handovers without an additional readback.

\section{THE UGLY}

The percentage of adverse events attributed to "communication" is suspiciously similar to "human error". Just as the label "human error" has been remarkably unproductive in improving patient safety, the label "communication" is not likely to provide much traction. If history is repeated, then the primary benefit of standardising handovers will be a new way to blame "sharp end" providers for failing to communicate critical information during the course of care. In the ugliest situation, idealised standards will be written that exclusively emphasise "safety" as an isolated goal. After-the-fact investigations will point to deviation from the standard as the main contributor to an undesired outcome, ignoring that trade-off decisions were made without the benefit of hindsight knowledge, that local actors always need to tailor plans of distant supervisors to a local situation, and that people adapt over time to feedback on how to trade off competing goals such as safety, productivity, profitability, satisfaction and quality of work life.

As we strive to steer clear of the ugly, another lesson might be gleaned from the NASA Johnson space shuttle mission control about how to update structured, yet flexible, standards over time. During the seventy-sixth space shuttle mission (STS-76), there was an anomalous auxiliary power unit (APU) leak detected during take-off. The official procedure at the time required aborting the mission and landing as soon as possible after achieving a stable orbiting configuration to reduce the risk of losing another of the three APUs, since it was judged that two APUs were required for a safe landing. After a series of debates, a decision was made to keep to the original schedule because, first, no additional risk was believed to be incurred because APUs were not used while in space, and, second, even a leaking APU might have some capability in an emergency landing. Even though the mission controllers were very busy dealing with other impacts of the anomaly, they chose to officially update the procedure during the mission. In this way, the controllers could verify that the decision makers would feel sufficiently comfortable with accepting this additional risk every single time the situation arose.

\section{Competing interests: None declared.}

The views expressed are solely those of the author and do not necessarily reflect the views of the Department of Veterans Affairs.

Oual Saf Health Care 2008;17:4-5.

doi:10.1136/qshc.2007.022772

\section{REFERENCES}

1. Patterson ES, Roth EM, Woods DD, et al. Handoff strategies in settings with high consequences for failure: lessons for health care operations. Int J Qual Health Care 2004;16:125-32.

2. Borowitz SM, Waggoner-Fountain LA, Bass EJ, et al. Adequacy of information transferred at resident signout (inhospital handover of care): a prospective survey. Qual Saf Health Care 2007;17:6-10.

3. Patterson ES, Woods DD, Cook RI, et al. Collaborative cross-checking to enhance resilience. Cognit Tech Work 2007;9:155-62.

4. Patterson ES. Voice loops: engineering overhearing to aid team coordination. In: Nemeth C, ed. Healthcare team communication. Hampshire, UK: Ashgate (in press).

5. Patterson ES, Woods DD. Shift changes, updates, and the on-call model in space shuttle mission control. Computer supported cooperative work. J Collab Comput 2001:10:317-46.

6. Woods DD, Hollnagel E. Joint cognitive systems: patterns in cognitive systems engineering. New York: Taylor \& Francis, 2006.

7. Hollnagel E. Human error: trick or treat? In: Durso F ed. Handbook of applied cognition. 2nd edn. New York: Wiley-Interscience, John Wiley \& Sons.

Access the latest content chosen by our Editors

BMJ Journals editors select an article from each issue to be made free online immediately on publication. Other material is free after 12 months to non-subscribers. Access the Editor's Choice from the home page - or expand your horizons and see what the other BMJ Journals editors have chosen by following the links on any BMJ Journal home page. 